\title{
Condiciones Sociales y de Salud de los Recicladores de Medellín
}

\section{Social conditions and health profile of recyclers from Medellín}

\author{
Jaime A. Gómez-Correa ${ }^{1}$, Andrés A. Agudelo-Suárez ${ }^{1,2}$ y Elena Ronda-Pérez² \\ 1 Facultad de Medicina, Facultad de Odontología. Universidad de Antioquia. Medellín, Colombia. \\ vicemed@gmail.com, agudeloandres@odontologia.udea.edu.co \\ 2 Área de Medicina Preventiva y Salud Pública. Universidad de Alicante, España. elena.ronda@ua.es
}

Recibido 7 Abril 2008/Enviado para Modificación 11 Octubre 2008/Aceptada 15 Noviembre 2008

\section{RESUMEN}

Objetivo Los recicladores en la ciudad de Medellín (Colombia) son un grupo social de gran tradición que en una gran mayoría han trabajado en sectores informales de la economía. Este estudio tiene como objetivo analizar la situación social y económica, el perfil de morbilidad y las condiciones de acceso a los servicios de salud en una población de recicladores y sus familias.

Métodos Estudio descriptivo, mediante encuestas de caracterización socioeconómica en recicladores y familiares (515 personas) y su valoración médica (174 personas). Se describieron las variables estudiadas por edad y sexo. El área de estudio estuvo constituida por los recicladores del sector de Guayaquil (Medellín).

Resultados El 71,6 \% de la población analizada habita en barrios de estratos medio bajo y bajo, en su mayoría población joven. Se establecen principalmente (83 \%) por uniones libres y matrimonios con un promedio de 5,3 $\pm 2,7$ personas por familia. El $42 \%$ viven en situación de pobreza. Se reportan infecciones respiratorias $(32,5 \%)$ y diarreicas $(10,5 \%)$ y en la valoración médica, se encuentran enfermedades crónicas $(47,1 \%)$, entre ellas enfermedades del sistema nervioso y órganos de los sentidos (20,1\%). El $37 \%$ no están cubiertos por un seguro de salud.

Conclusiones Se presentan condiciones de vida y de salud deficientes, que requieren soluciones concretas para este grupo que permitan el mejoramiento del nivel social, la generación de empleo, el aumento de la cobertura en salud y la realización de campañas de promoción de la salud y prevención de la enfermedad.

Palabras Clave: Condiciones sociales, perfil de salud, accesibilidad a los servicios de salud, poblaciones vulnerables (fuente: DeCS, BIREME).

\section{ABSTRACT}

Objective Recyclers in the city of Medellin (Colombia) are social groups having a long-standing tradition; many of them have worked in informal economy sectors. This study was aimed at analysing their economic and social situation, health profile 
and health service accessibility (i.e. for a recycler group and their families).

Methods A descriptive study was carried out by means of socioeconomic characterisation surveys of recyclers and relatives (515) and 174 medical examinations. The variables were described by age and gender. The area being studied was the locality of Guayaquil (Medellín) and its recyclers who accepted participating in the study.

Results $71,6 \%$ of the population being analysed inhabited lower and middle-lower class neighbourhoods; most were young people. The main household organisation consisted of living with a partner and marriage (83\%), having 5,3 $\pm 2,7$ people per family. $42 \%$ were living in conditions of poverty (1-2 dollars per day income). Respiratory (32,5\%) and diarrheic infections (10,5\%) were reported. Medical examination revealed chronic diseases $(47,1 \%)$, nervous system and sense organ diseases $(20,1 \%) .37 \%$ had no health insurance.

Conclusion The recyclers and their relatives were living in deficient social conditions. Public health action for the benefit of this group is required by means of generating employment, increasing health insurance cover and promoting specific health promotion and disease prevention strategies.

Key Words: Social conditions, health profile, health services accessibility, vulnerable populations (source: MeSH, NLM).

$\mathrm{L}$ a sociedad moderna ha desarrollado un modelo económico con alto consumo de bienes y con la subsiguiente producción de desechos que ocasionan gran impacto ambiental. En un informe sobre el desarrollo mundial del 2003, se señala que algunas ciudades europeas con más de un millón de habitantes pueden generar hasta 1600 toneladas de residuos sólidos por día (1); esto también se cumple en una ciudad como Medellín donde se estimó una producción de 1545 toneladas por día en el año 2003 (2). Esta problemática exige el desarrollo de respuestas que permitan su manejo, como por ejemplo el reciclaje (3).

Esta alternativa, prolifera en otros países subdesarrollados y en vía de desarrollo (3), como una forma de trabajo y manutención (4). En el caso colombiano, el reciclaje es ejercido por personas que en su mayoría se encuentran en el nivel informal de la economía. Se conocen comúnmente como recicladores. Esta labor es de gran importancia social, beneficia a sectores industriales y residenciales, disminuyendo los costos de producción de basura. Los recicladores recuperan del 10 al $12 \%$ del total de residuos sólidos producidos, equivalentes a 70 000 toneladas/año aproximadamente; esto representa un ahorro para la ciudad de Medellín de cerca de 5000 millones de pesos al año (2). Se estima que unas 280000 personas viven de las basuras y del reciclaje (2).

Existen algunos estudios que caracterizan la situación de salud de los recicladores. A nivel nacional, se realizó un diagnóstico de la situación 
socioeconómica y de salud por la Corporación Salud y Desarrollo en 1995(5); y se encontró otro estudio antropológico y social en indígenas Guahíbos en la ciudad de Bogotá (6). A nivel internacional, investigaciones en México, Brasil y Estados Unidos recogen las características particulares en cada país $(4,7,8)$.

La identificación de los determinantes que afectan las condiciones de vida en grupos vulnerables como los recicladores y sus familias, permite la generación de estrategias que respondan a sus problemas más inmediatos; tanto desde los servicios sanitarios, como desde políticas sociales y económicas $(9,10)$. Hasta el momento, poco se conoce de la realidad local en estos colectivos en la ciudad de Medellín.

En este marco surgió la necesidad de conformar organizaciones para mejorar las condiciones de trabajo de estas personas. Así, la Asociación de Ingenieros Sanitarios de Antioquia (AINSA), diseñó un proyecto en el que su primer objetivo, estaba encaminado a conocer las condiciones sociales y de salud de los recicladores que trabajan en el Sector de Guayaquil (Medellín). En un primer análisis se identificaron las condiciones de trabajo específicas de los recicladores, y su impacto sobre la salud (11). El objetivo de este segundo estudio fue analizar las condiciones sociales y de salud de la población de recicladores en Medellín y sus familias, por medio de la caracterización de la situación laboral, social y económica, el perfil de morbilidad y sus condiciones de acceso a servicios de salud.

\section{MÉTODOS}

Se trata de un estudio descriptivo que incluye encuestas socioeconómicas, y valoraciones médicas. La población de estudio fue extraída a partir del censo de recicladores informales del Sector de Guayaquil, registrados en una base de datos de AINSA. Luego de organizar los datos y revisar inconsistencias, se llevaron a cabo contactos telefónicos. Finalmente, fueron seleccionados 97 recicladores y sus familias (515 personas en total), quienes voluntariamente aceptaron participar en la investigación.

El estudio estuvo conformado en dos fases: En la primera, 8 estudiantes de medicina previamente entrenados realizaron una visita al domicilio de los 97 recicladores, aplicando una encuesta. Se recopiló información sobre aspectos demográficos del reciclador y de su familia; condiciones laborales, variables socio-económicas; características generales de la vivienda, aspectos generales de la salud (morbilidad percibida, vacunación y estrategias de atención en 
salud) y afiliación al Sistema General de Seguridad Social en Salud. En una segunda fase se realizaron valoraciones médicas, por parte de 3 médicos titulados. El examen constaba de anamnesis y una exploración clínica. Se realizó a 174 personas (49 recicladores y 125 familiares) que voluntariamente asistieron a una jornada de salud programada.

Los datos fueron analizados estadísticamente por medio del programa SPSS versión 11.0. Se realizó un análisis descriptivo para cada una de las variables estudiadas, estableciendo diferencias por edad y por sexo en la población encuestada. Por último, se realizaron pruebas Chi cuadrado para variables cualitativas.

También se construyeron indicadores como el de Línea de Pobreza; éste se optó por medir según el Banco Mundial (1). Todo hogar con un ingreso diario $£$ a 2 dólares se consideró en estado de pobreza; todo hogar con un ingreso diario $£ 1$ dólar se consideró en estado de indigencia. De otra parte, el indicador de Necesidades Básicas Insatisfechas, se construyó a partir de evaluar la calidad de la vivienda, hacinamiento crítico, servicios públicos, deserción escolar y alta dependencia económica. Este indicador permite clasificar la población en pobreza a aquellos hogares que tienen una necesidad básica insatisfecha y en miseria a aquellos que tienen dos ó más necesidades de las anteriormente mencionadas $(12,13)$. Por ultimo, la tasa de alfabetismo, se definió como el porcentaje de personas de 15 años o más que pueden leer, escribir y comprender un texto corto y sencillo sobre su vida cotidiana (14).

Se contó con el consentimiento informado para la realización del estudio; se aclararon en conjunto las limitaciones del mismo, y se garantizó la confidencialidad de los datos.

\section{RESULTADOS}

Aspectos sociodemográficos y condiciones de vida

Esta población de recicladores y familiares presenta un promedio de 5,3 \pm 2,7 personas por familia; se trata de una población joven, en la cual el 58,1 \% es menor de 20 años. El 72,2 \% viven en zonas que se caracterizan por ser de estratos socioeconómicos medio bajo y bajo (Zonas Nororiental, Centro-oriental y Centro-occidental). La relación de pareja se establece principalmente mediante la unión libre y el matrimonio, conformando tipologías familiares en las que prevalece la familia nuclear (Tabla 1 ). 
Más de la mitad de las personas de 18 años o mayores no tienen ninguna escolaridad o tienen la primaria incompleta. La tasa de alfabetismo es del 83,5 $\%$. El 29,7 \% del total de integrantes de la familia son recicladores (en su mayoría hombres). La mayoría de los hogares tiene un ingreso menor a dos dólares. Más de la mitad de los hogares se encuentran en pobreza y en miseria (Tabla 2).

Tabla 1. Características sociodemográficas de la población de recicladores y familiares incluidos en el estudio (Medellín)

\begin{tabular}{|c|c|c|c|c|c|c|c|}
\hline & \multicolumn{2}{|c|}{ Hombres } & \multicolumn{2}{|c|}{ Mujeres } & \multicolumn{2}{|c|}{ Total } & \multirow{2}{*}{$p^{*}$} \\
\hline & $n$ & $\%$ & $\mathrm{n}$ & $\%$ & $\mathrm{n}$ & $\%$ & \\
\hline Sexo & 251 & $4 B, 7$ & 264 & 51,3 & 515 & 100 & $m$ \\
\hline \multicolumn{8}{|l|}{ Lugar de Residencia } \\
\hline Zona Nor-Oriental & 106 & 42,2 & 116 & 43,9 & 222 & 43,1 & \\
\hline Zona Nor- Occidental & 25 & 10,0 & 23 & 8,7 & 48 & 9,3 & \\
\hline Zona Centro- Oriental & 39 & 15,5 & 39 & 14,8 & 78 & 15,1 & \\
\hline Zona Centro-Occidental & 36 & 14,4 & 36 & 13,6 & 72 & 14,0 & \\
\hline Zona Sur- Occidental & 11 & 4,4 & 10 & 3,8 & 21 & 4,1 & \\
\hline Otras & 34 & 13,5 & 40 & 15,2 & 74 & 14,4 & 0.9807 \\
\hline \multicolumn{8}{|c|}{$\begin{array}{l}\text { Ocupación de los miembros de la } \\
\text { familia }\end{array}$} \\
\hline Reciclador & 106 & 42,2 & 47 & 17.8 & 153 & 29,7 & \\
\hline Estudiante & 53 & 21,1 & 72 & 27,3 & 125 & 24,3 & \\
\hline Menor de edad ( $<7$ años) & 36 & 14,3 & 40 & 15,2 & 76 & 14,8 & \\
\hline Ama de casa & 0 & 0,0 & 61 & 23.1 & 61 & 11,8 & \\
\hline Desempleado & 30 & 12,0 & 17 & 6,4 & 47 & 9,1 & \\
\hline Trabajador informal & 13 & 5,2 & 12 & 4,5 & 25 & 4,9 & \\
\hline Obrero & 6 & 2,4 & 1 & 0.4 & 7 & 1,4 & \\
\hline Otros (no clasificados) & 7 & 2,8 & 14 & 5,3 & 21 & 4,1 & $<0.0001$ \\
\hline \multicolumn{8}{|c|}{ Nivel educativo $(\geq 18$ afios) $n=275$} \\
\hline Total & 133 & 100 & 142 & 100 & 275 & 100 & \\
\hline Ninguno & 16 & 12,0 & 20 & 14,1 & 36 & 13,1 & \\
\hline Primaria Incompleta & 49 & 36,8 & 60 & 42,3 & 109 & 39,6 & \\
\hline Primaria Completa & 31 & 23,3 & 26 & 18,3 & 57 & 20,7 & \\
\hline Secundaria Incompleta & 22 & 16,5 & 25 & 17,6 & 47 & 17,1 & \\
\hline Secundaria Completa & 11 & 8,3 & 9 & 6,3 & 20 & 7,3 & \\
\hline Otros & 2 & 1,5 & 2 & 1,4 & 4 & 1,5 & \\
\hline Sin dato & 2 & 1,5 & 0 & 0,0 & 2 & 0,7 & 0,6639 \\
\hline \multicolumn{8}{|l|}{ Tipo de familia ( $n=97$ familias) } \\
\hline Nuclear & & & & & 33 & 34 & \\
\hline Extensa & & & & & 29 & 29,9 & \\
\hline Otros tipos & & & & & 17 & 17,5 & \\
\hline Monoparental & & & & & 9 & 9,3 & \\
\hline Moderna & & & & & 6 & 6,2 & \\
\hline Vive Solo & & & & & 3 & 3,1 & - \\
\hline
\end{tabular}

Condiciones de salud

En relación a la cobertura de vacunación contemplada en el Programa Ampliado de Inmunizaciones para Colombia (PAI), se observó que el 39 \% de los niños menores de 5 años no tenían el carné de vacunación. Un 75 \% tenían el esque- 
ma incompleto del PAI (Tabla 3). Al evaluar el estado de vacunación con TD en mayores de 7 años, se observa que solo el $4 \%$ tiene el esquema completo.

Tabla 2. Componentes e Indicador de Necesidades Básicas Insatisfechas en la población de recicladores y sus familias (Medellín) $n=97$ hogares

\begin{tabular}{|c|c|c|}
\hline Componentes Necesidades Básicas Insatisfechas & $\mathrm{n}$ & $\%$ \\
\hline Calidad de la vivienda adecuada & 75 & 77,3 \\
\hline Servicios Públicos adecuados (Agua, luz, alcantarillado) & 77 & 79,0 \\
\hline Sin Hacinamiento en los hogares & 81 & 83,5 \\
\hline Sin ausentismo escolar & 87 & 89,6 \\
\hline Sin dependencia económica & 70 & 72,2 \\
\hline Sin Necesidades Básicas Insatisfechas & 43 & 44,3 \\
\hline Pobreza & 27 & 27,8 \\
\hline Miseria & 27 & 27,8 \\
\hline
\end{tabular}

Tabla 3. Esquema de vacunación para los biológicos del Programa Ampliado de Inmunizaciones (PAI) en hijos de recicladores menores de 5 años (Medellín) n=64

\begin{tabular}{lcccc}
\hline \multirow{2}{*}{ Vacuna } & \multicolumn{2}{c}{ Completa } & \multicolumn{2}{c}{ Incompleta } \\
& $\mathrm{N}$ & $\%$ & $\mathrm{~N}$ & $\%$ \\
\hline TBC & 32 & 50,0 & 32 & 50,0 \\
DPT & 28 & 43,8 & 36 & 56,3 \\
Polio & 26 & 40,6 & 38 & 59,4 \\
MMR & 28 & 43,8 & 36 & 56,3 \\
Haemophilus & 19 & 29,7 & 45 & 70,3 \\
Hepatitis & 32 & 50,0 & 32 & 50,0 \\
Total Biológicos & 16 & 25,0 & 48 & 75,0 \\
\hline
\end{tabular}

En cuanto a la morbilidad percibida evaluada mediante la pregunta “ ¿Se ha sentido enfermo en los 15 días anteriores a la encuesta?", el 38,8 \% reportó alguna enfermedad en los últimos quince días. El 64,5\% hacen acciones diferentes a la de consultar al sistema sanitario (Tabla 4). Estas personas lo hacen principalmente por falta de dinero o por considerar que la enfermedad no es grave y es innecesaria la consulta médica. Los diagnósticos reportados por la población que sufrió alguna enfermedad en los últimos 15 días son: infección respiratoria aguda (más frecuente en hombres), enfermedad diarreica aguda (más frecuente en mujeres) (Tabla 4).

Teniendo en cuenta las variables antropométricas la estatura promedio de las mujeres $\geq 18$ años es de $1,52 \mathrm{~m}$ (1,39 m-1,68 m) y de los hombres $\geq 18$ años es de en 1,63 m (1,48 m-1,75 m). Según el índice de Masa Corporal (IMC), el $27,7 \%$ se encontró en sobrepeso y el 5,3 \% con bajo peso. Los hombres presentaron una proporción más baja de sobrepeso (14,6 \%) y de bajo peso (4,9 \%) con respecto a las mujeres (37,7 \% y 5,7 \% respectivamente). La descripción antropométrica en los menores de 18 años se realizó mediante los 
indicadores de desnutrición aguda (peso para la estatura), desnutrición global (peso para la edad) y desnutrición crónica (estatura para la edad). Se encontró un 22,1 \% de déficit en el peso para la edad, y un 46,3 \% de déficit de estatura para la edad.

Tabla 4. Condiciones de salud de los recicladores y sus familias. Medellín

\begin{tabular}{|c|c|c|c|c|c|c|c|}
\hline \multirow{2}{*}{ Caracteristica } & \multicolumn{2}{|c|}{ Hombres } & \multicolumn{2}{|c|}{ Mujeres } & \multicolumn{2}{|c|}{ Total } & \multirow{2}{*}{$p^{\prime \prime}$} \\
\hline & $n$ & $\%$ & $\mathrm{~N}$ & $\%$ & $\mathrm{~N}$ & $\%$ & \\
\hline \multicolumn{8}{|l|}{ Enfermedad en los últimos 15 dias ( $n=515)$} \\
\hline $\mathrm{Si}$ & 89 & 35,5 & 111 & 42,1 & 200 & 38,8 & \multirow{3}{*}{0,1923} \\
\hline No & 161 & 64,1 & 153 & 57,9 & 314 & 61,0 & \\
\hline NSINR & 1 & 0,4 & 0 & 0.0 & 1 & 0.2 & \\
\hline \multicolumn{7}{|l|}{ Acciones realizadas $(n=200)$} & \multirow{8}{*}{0,8414} \\
\hline Ir al médico & 28 & 31,5 & 36 & 32,4 & 64 & 32,0 & \\
\hline Tomar remedios caseros & 26 & 29,2 & 36 & 32,4 & 62 & 31,0 & \\
\hline Automedicarse & 12 & 13,5 & 17 & 15,3 & 29 & 14,5 & \\
\hline Consultar al farmaceutico & 2 & 2,3 & 5 & 4,5 & 7 & 3,5 & \\
\hline Consultar familiar & 2 & 2,3 & 1 & 0.9 & 3 & 1,5 & \\
\hline No hacer nada & 9 & 10,1 & 8 & 7,2 & 17 & 8,5 & \\
\hline Otras & 10 & 11,1 & 8 & 7.2 & 18 & 9,0 & \\
\hline \multicolumn{7}{|l|}{ Morbilidad percibida $(n=200)^{p}$} & \multirow{9}{*}{0,1398} \\
\hline Infección Respiratoria & 35 & 39,3 & 30 & 27,0 & 65 & 32,5 & \\
\hline Enfermedad Diarreica & 9 & 10,1 & 12 & 10,8 & 21 & 10,5 & \\
\hline Eruptiva Febril & 1 & 1,1 & 2 & 1,8 & 3 & 1,5 & \\
\hline Enfermedades de la piel & 5 & 5,6 & 2 & 1,8 & 7 & 3,5 & \\
\hline Enfermedades de los dientes & 2 & 2,3 & 0 & 0,0 & 2 & 1,0 & \\
\hline Accidentes & 2 & 2,3 & 1 & 0,9 & 3 & 1,5 & \\
\hline Otras no especificadas & 23 & 25,8 & 42 & 37,8 & 65 & 32,5 & \\
\hline Reportan varios sintomas-o enfermedades & 12 & 13,5 & 22 & 19.8 & 34 & 17,0 & \\
\hline \multicolumn{7}{|l|}{ Valoracion Médica $(n=174)^{6}$} & \multirow{14}{*}{0,2583} \\
\hline Sano & 11 & 13,1 & 13 & 14,4 & 24 & 13,8 & \\
\hline $\begin{array}{l}\text { Enfermedades del sistema nervioso y de los } \\
\text { organos de los sentidos }\end{array}$ & 19 & 22,6 & 16 & 17,8 & 35 & 20,1 & \\
\hline $\begin{array}{l}\text { Enfermedades endocrinas, nutrición, } \\
\text { metabolismo e inmunes }\end{array}$ & 9 & 10,7 & 12 & 13,3 & 21 & 12,1 & \\
\hline Enfermedades del sistema respiratorio & 10 & 11,9 & 6 & 6,7 & 16 & 9,2 & \\
\hline Enfermedades del sistema circulatorio & 5 & 5,9 & 10 & 11,1 & 15 & 8,6 & \\
\hline Enfermedades del sistema geniltourinario & 5 & 5,9 & 8 & 8.9 & 13 & 7,5 & \\
\hline Enfermedades del sistema digestivo & 5 & 5,9 & 5 & 5,6 & 10 & 5,8 & \\
\hline Alteraciones osteo- musculares & 4 & 4,8 & 3 & 3,3 & 7 & 4,0 & \\
\hline Enfermedades infecciosas y parasitarias & 2 & 2,4 & 4 & 4,4 & 6 & 3,5 & \\
\hline Enfermedades de la piel & 4 & 4,8 & 1 & 1,1 & 5 & 2,8 & \\
\hline Enfermedades de los dientes & 3 & 3,6 & 2 & 2,2 & 5 & 2,8 & \\
\hline Trastornos mentales & 4 & 4,8 & 0 & 0,0 & 4 & 2,3 & \\
\hline Otras & 3 & 3.6 & 10 & 11,1 & 13 & 7.5 & \\
\hline
\end{tabular}

a Prueba de sianifosción Chi cuadrado de Pearson; b Claseficado de acuerdo con la CIE-9

Atendiendo a la Clasificación Internacional de Enfermedades (CIE 9), se encuentran en primer lugar las enfermedades del sistema nervioso y órganos de los sentidos con un 20,1\%; dentro de este grupos sobresalen problemas de disminución de la agudeza visual y las cataratas; le siguen en importancia las 
enfermedades endocrinas, de la nutrición, metabolismo y trastornos inmunes con en un 12,1\%; en este grupo se destacan las enfermedades relacionadas con la desnutrición y la anemia (Tabla 4).

Comparando la morbilidad autopercibida y la morbilidad por diagnóstico clínico el 61 \% de la población estudiada se percibió como sana en los últimos 15 días; comparado con un 13,8 \% de personas sanas por medio de examen. Además la población percibió en mayor proporción las enfermedades de tipo infeccioso con respecto a las enfermedades de tipo crónico diagnosticadas por la valoración médica (enfermedades de los órganos de los sentidos, endocrinas, sistema circulatorio, mentales y de los dientes) (Tabla 4).

Por último, sobre la cobertura en el Sistema General de Seguridad Social en Salud Colombiano, el 59 \% del total de este grupo poblacional se encuentra en el régimen subsidiado y el $37 \%$ son vinculados.

\section{DISCUSIÓN}

Los resultados de este estudio muestran como los recicladores y sus familias, conforman un grupo social que reside principalmente en barrios periféricos de la ciudad y en estratos socioeconómicos más bajos. Se encontraron dificultades en la vivienda, bajo nivel educativo, problemas de acceso a servicios de salud y morbilidad de tipo infeccioso y crónico en comparación con otras zonas de la ciudad de Medellín (15). Estos hechos tienen repercusiones en los perfiles de morbilidad de los grupos sociales más desfavorecidos $(16,17)$.

Las difíciles condiciones de trabajo, y la insuficiente calidad de vida de la población se materializan en una estructura corporal limitada, con un promedio de estatura bajo, presencia de desnutrición crónica en menores y un IMC con tendencia al sobrepeso especialmente en mujeres como consecuencia de consumos alimentarios inapropiados $(18,19)$. Esto representa un reto para la salud pública, puesto que se están presentando problemas de sobrepeso y obesidad en países de menor capacidad económica $(20,21)$.

El nivel de morbilidad hallado en la población estudiada por la valoración médica es mayor que el reportado en la morbilidad sentida, lo cual hace pensar en una baja auto percepción de la enfermedad y cierto estoicismo producto de condiciones continuas que pueden sentirse como normales. Los problemas encontrados, en algunos casos, dependen de los factores de riesgo a los cuales se exponen diariamente en su labor de recicladores de basuras (11), y las 
condiciones de vida (16), que los colocan en una situación de vulnerabilidad como en otros grupos sociales (22), y con respecto a la población colombiana (23). Sin embargo, los datos encontrados deben interpretarse con cautela, teniendo en cuenta el contexto local de la población a estudio.

En cuanto a la cobertura del sistema sanitario en Colombia, se observaron problemas de acceso a los servicios de salud, y barreras sociales, geográficas, económicas, entre otras, que en la práctica impiden la solución a los problemas de salud de la población más vulnerable (24).

Con respecto a las limitaciones del estudio cabe mencionar la no posibilidad de hacer inferencias en la población general, debido a la dificultad de hacer un muestreo representativo de los recicladores de la ciudad de Medellín y por la voluntariedad de la participación del estudio. Esta investigación, es una exploración inicial en un grupo poblacional específico, pero que puede constituir una guía para estudios de mayor alcance y profundidad, que evidencien las condiciones sociales y su impacto en la salud en grupos vulnerables como el estudiado, con técnicas mixtas (cuantitativas y cualitativas). Otro elemento a considerar, sería las diferencias encontradas por género, que evidencian la necesidad de explorar con mayor profundidad las características laborales y sociales de ambos grupos.

El Estado Colombiano, con la colaboración de otros sectores sociales y de la economía, debe contribuir a la generación de políticas sociales, en salud y laborales, que permitan a los colectivos vulnerables, tener facilidades para la conformación de empresas, la solución de las barreras de acceso a los servicios de salud, y la asesoría técnica necesaria para que puedan tener reconocimiento social como un grupo que le presta grandes servicios a la ciudad *

Agradecimientos. A los estudiantes de medicina y a los médicos que participaron en la recolección de la información. A los auxiliares de investigación Alexandra Restrepo y Oscar Carmona que apoyaron el análisis de los datos, a Juan Ignacio Sarmiento por su asesoría estadística y a los recicladores y sus familias que participaron en el estudio, permitiendo investigar una realidad social importante y poco conocida. Financiación: Convenio entre la Asociación de Ingenieros Sanitarios de Antioquia -AINSA- y la Facultad de Medicina de la Universidad de Antioquia. 


\section{REFERENCIAS}

1. Banco Mundial. Informe sobre el desarrollo mundial 2003. Desarrollo sostenible en un mundo dinámico. Bogota: Multiprensa libros y alfa omega; 2003.

2. Sepúlveda LA. Organización y dignificación de recicladores informales. El reto. 2003; 48: 23-35 3. Medina M. Reciclaje de residuos sólidos en América Latina. Frontera Norte. 1999; 11 (21): 7-31.

4. Long WA. La recuperación informal de residuos sólidos en Guadalajara: Una Investigación del conflicto entre los pepenadotes y la economía informal de los desechos. Ecología Política (España) 2000; 19: 13-20.

5. González PC, Ochoa D, Duarte MB, Zarate MA, Alcaraz FG y Castro AL. Los Recicladores en Santa Fe de Bogotá. Bogotá: Corporación Salud y Desarrollo; 1996.

6. Ortiz B. Los nuevos recolectores: Los Guahíbos recicladores de basura en Ciudad Bolívar. Boletín Antropológico. 2002; 20 (54): 483-498.

7. Rendleman N, Feldstein A. Occupational Injuries among Urban Recyclers. J Occup Environ Med. 1997; 39(7):672-5.

8. Carvalho BHM. Cooperativismo y Reciclado. Estrategias de Supervivencia de los seleccionadores de basura de Salvador, Bahía, Brasil. Scripta Nova. [Internet] Revista Electrónica de Geografía y Ciencias Sociales. 1999: 45; 18 Disponible en: http://www.ub.es/geocrit/ sn-45-18.htm Consultado 20 de Febrero de 2008.

9. Castellanos P. Los modelos explicativos del proceso salud-enfermedad. Los determinantes sociales. En: Martínez F. Salud Pública. Madrid; Mc Graw Hill- Interamericana: 1998.

10. Castellanos PL. Evaluación de la situación de salud y sus tendencias en grupos de población. En: II Taller Latinoamericano de Medicina Social. Caracas-Venezuela: Asociación Latinoamericana de Medicina Social; 1991.

11. Gómez-Correa JA, Agudelo-Suárez AA, Sarmiento-Gutiérrez JI, Ronda-Pérez E. Condiciones de trabajo y salud de los recicladores urbanos de Medellín (Colombia). Arch Prev Riesgos Lab 2007; 10 (4): 181-187.

12. Muñoz M. Los indicadores de pobreza utilizados en Colombia: una crítica. En: Cinep. Inserción precaria, desigualdad y elección social. Bogotá; Antrophos Ltda.; 2000.

13. Departamento Administrativo Nacional de Estadística. Metodología de la encuesta Nacional de hogares. Colombia. Bogotá: DANE; 1985.

14. Programa de las Naciones Unidas para el desarrollo. Informe Sobre Desarrollo Humano. México: PNUD; 1995.

15. Alcaldía de Medellín. Encuesta de Calidad de Vida 2004. Medellín: Alcaldía de Medellín; 2004.

16. Echeverri LE. La salud en Colombia: Abriendo el siglo... y la brecha de las inequidades. Rev Gerenc políticas salud. 2002; 1(3):76-94.

17. Leon DA, Walt G. International perspectives on health inequalities and policy. BMJ 2001; 322(7286): 591-594.

18. Barria RM, Amigo, H. Transición Nutricional: una revisión del perfil latinoamericano. Arch. latinoam nutr. 2006; 56 (1): 3-11.

19. Rivera JA, Barquera S, González-Cossio T, Olaiz G, Sepúlveda J. Nutrition transition in Mexico and in other Latin American countries. Nutr Rev. 2004; 62 (7 Pt 2): S149-S157

20. Popkin BM, Doak CM. The obesity epidemia is a worldwide phenomenon. Nutr Rev. 1998; 56:106-14.

21. Peña M, Bacallao J (eds). La obesidad en la pobreza. Un nuevo reto para la salud pública. Washington, D.C: Organización Panamericana de la Salud; 2000. Publicación Científica 576.

22. Cáceres DC, Izquierdo VF, Mantilla L, Jara J, Velandia M. Perfil epidemiológico de la población desplazada por el conflicto armado interno del país en un barrio de Cartagena, Colombia, 2000. Biomédica (Bogotá) 2002; 22 (supl.2): 425-444.

23. Profamilia. Salud sexual y reproductiva en Colombia: resultados encuesta nacional de demografía y salud 2000. Bogotá: Profamilia; 2000.

24. Herazo AB. Panorama socioeconómico y político de la salud en Colombia. Rev Fed Odontol Colomb 2002;(201):95-100. 